\section{Letter: Peas, Priorities and Primary Care: Cultural competency starts at home.}

\section{To the Editor:}

On January 27 and 28, 2005, I attended my first annual meeting of the National Coalition for Health Professional Education in Genetics (NCHPEG). The other attendees were from diverse academic, governmental, and industry settings and shared a common goal of promoting the importance of genetic issues in health care. As a practicing primary care physician in the United States with an interest in the role of genetics in primary care and a Ph.D. in Human Genetics, I found the presentations enlightening, as well as disturbing.

"Focus on Family History" was the theme for this year's meeting, a topic garnering national attention since the unveiling of the Family History Initiative by the U.S. Surgeon General last November. ${ }^{1}$ The basic idea of the initiative is to elevate public awareness of the role family history plays in determining risk for many common disorders. An increase in awareness is hoped to spur individuals to gather their family histories and to motivate them to seek their physician's aid in mitigating disease risk. A secondary intent is to stimulate clinicians' interest in the role of genetics in their practices. The impetus for this initiative is very reasonable: several disorders of major public health impact have a clear genetic component (for example, coronary heart disease, colon cancer, breast cancer, and diabetes) and early identification of elevated risk may permit early intervention and reduction of subsequent disease risk.

The content of the meeting left no doubt that the primary care community is ill prepared to deal with genetics issues. It was striking that there was also an undercurrent of incredulity among meeting attendees and presenters that primary care physicians don't see the clear need (at least from the geneticists' perspective) to become better versed in the health improvement tools that the genetics community has to offer. Several speakers presented rigorously gathered data regarding the barriers the genetics community faces in getting their message to primary care. Identified barriers might be distilled to three central themes: lack of genetics knowledge and resources, lack of perception of the relevance of genetics to daily practice, and lack of time in a busy office setting.

I propose that a fourth overarching barrier must be overcome before the genetics community will have its voice meaningfully heard in primary care. This barrier is, to borrow a popular term in academic medicine, a lack of cultural competency. Predictably, the genetics community views, describes, and interacts with the world through a lens of genetics. This lens distorts the geneticists' perspective on priorities in primary care, and results in a presentation of genetics priorities in a language and "cultural context" that primary care physicians don't understand. As with issues between ethnic, racial, and cultural groups, a deadly trap must be avoided by both genetics and primary care communities, the lack of immediate adoption of each other's priorities and values does not connote ignorance and lack of sophistication.
One particular presentation had examples of how easily well intentioned research designed to bring genetics and primary care closer together might actually worsen the situation. The presenter shared preliminary data from interviews of rural, suburban, and urban primary care physicians regarding the role of genetics in their practice. In addition to aggregate data from carefully analyzed interview transcripts, the speaker also presented quotes from practicing doctors. Several of these quotes brought gasps of horror from attendees, ostensibly because they found them hopelessly naive in the age of genomics. The audience reaction to two quotes in particular drove home the fact that the trap of assuming ignorance without understanding culture looms large. I will paraphrase the quotes. First, a provider stated that he was not interested in being an early adopter of genetics tests for familial cancer syndromes because he did not want to be the first one on the block offering a new test. Second, a provider was quoted as saying that he thought there was genetic testing available for breast cancer syndromes, and that he vaguely remembered that there might be similar testing available for familial colon cancer syndromes. As these were direct quotes from a phone interview, the language was not very formal, further engendering a feeling that the physicians were less than sophisticated (much as happens with certain politicians).

As a primary care physician, I feel compelled to examine these two quotes in more detail, flushing out the cultural context in which they may have been made. First, take the quote regarding not wanting to be an early adopter of genetic testing and technology. To the geneticist, tests for hereditary breast and ovarian cancer syndrome and the familial colon cancer syndromes have been around for quite some time (about a decade), have reasonable sensitivities and specificities, have fair predictive values, and have ample evidence supporting improved outcomes for their use (typically studies of interventions in mutation positive individuals in families with high burdens of disease). The primary care physician, on considering these tests, may recall the story of prostate-specific antigen (PSA) testing. PSA testing has been around for decades, was originally (and still is) promoted by a specialty community as having an acceptably high sensitivity and specificity, and had some level of evidence that outcomes might be improved through its use (detecting and curing early prostate cancer). Years and many studies later, the utility of this test remains an extensive source of debate in the primary care community, and is not supported as an essential part of routinely offered health care. $^{2,3}$ Given that the promotion of cancer genetics testing has not been presented in the language of evidence-based medicine to those in primary care, it is no wonder that many are reluctant to quickly assimilate these technologies into their practices.

Second, consider the quote relating a less than confident recollection of the availability of testing for breast and colon cancer syndromes. To the academic geneticist, the quoted individual must seem perilously out of date. As a primary care provider, I propose that the lack of knowledge (and the lack of interest in the topic it suggests) is less a product of neglect and 
ignorance, and more an issue of competing priorities in primary care. In most primary care settings, days are long, patients (and paperwork) are numerous, and less than ideal tradeoffs between what one can do to promote health and what is reasonably possible to do are plentiful. ${ }^{4,5}$

To illustrate why the familial cancer syndromes may not be on the mind of the quoted primary care physician as he walks into a yearly health maintenance visit, consider the following analysis. Assume that in an unselected population, the colon and breast cancer syndromes for which we have testing have an aggregate prevalence of $1 / 200$. Now consider that the average family physician has a patient panel size of roughly 2,500 individuals. If we neglect the fact that some of these patients are likely too old or too young to benefit from looking for a family history of a breast or colorectal cancer syndrome, this translates to about 13 potentially affected individuals. Now consider that the primary care physician recognizes those at-risk individuals using either screening criteria (that are not universally agreed upon in the genetics community), or because the patients arrive in the office with detailed family histories demonstrating risk. The physician effectively counsels the at-risk individuals on their testing options. Assume half (7) agree to, can pay for, and qualify for testing (some likely do not have surviving or available affected relatives). Subsequent testing identifies mutations putting half (4) of these individuals at an elevated (but, again, not universally agreed upon) risk. Now assume that the primary care physician counsels these 4 individuals on their treatment options (including increased surveillance, chemoprophylaxis, and prophylactic surgeries). For many of these interventions, there is not irrefutable evidence demonstrating a reduction of morbidity and mortality in patients ascertained from the general population. Perhaps half (2) of these individuals will agree to, be able to afford, and be candidates for the interventions. Many hours and healthcare dollars later, we are down to 2 patients that might benefit from family history screening for familial cancer syndromes.

Now consider smoking. Assume a patient panel size of 2,500 as well as a smoking prevalence of $20 \%$. Again, neglect that some are too young to smoke (but are probably never too young to be counseled on smoking); one is never too old to stop smoking and gain some benefit (at least economic). This suggests that the average primary care physician's panel contains about 500 smokers. Now assume that at yearly visits, the physician is able to convince about 20\% (100) of these individuals to try one of the proven approaches to smoking cessation, which are essentially universally covered by private as well as public payers. Of these 100 , about 30 who attempt cessation with help are smoke-free at one year. For these 30, there is irrefutable evidence that smoking cessation prolongs and improves life. Fifteen times as many patients benefit, and the total cost in both time and dollars spent per individual is likely a fraction of that for the interventions for the hereditary cancer syndromes.

Does it remain as obvious that the quoted primary care physician is hopelessly behind the times? Though one might quibble with some of the assumptions made in the analysis, the logic is still valid if one assumes that $100 \%$ of the persons at risk for the cancer syndromes agree to testing, have mutations, and accept all currently recommended interventions. A similar analysis might be done for any genetic disorder with low prevalence (the definition of which primary care and genetics communities might not immediately agree on) that requires expensive testing, extensive primary care physician knowledge, and extensive office time to help the patient deal with the incredibly complex issues testing raises.

I present these points not because I am a nihilist regarding the era of genomics.

On the contrary, I am extremely excited to be in a front row seat as the promise of genetics unfolds. I raise these issues to stimulate the genetics community to learn to "think primary care" before they spend significantly more time, money, and good intellectual effort on getting primary care doctors to "think genetic". I also call on primary care physicians to become active contributors to the development of medical genetics through organizations such as NCHPEG and professional medical societies. Historically, the melding of cultures has yielded spectacular benefits, and a balanced blending of the genetics and primary care communities holds great promise to revolutionize medicine.

\section{W. Gregory Feero, $\mathrm{MD}, \mathrm{PhD}$ Maine-Dartmouth Family Practice Residency Program Fairfield, ME}

\section{References}

1. Guttmacher AE, Collins FS, Carmona RH. The family history-more important than ever. N Engl J Med 2004;351:2333-2336.

2. Screening for prostate cancer: recommendations and rationale. www.guideline.gov. Accessed January 30, 2005. United States Preventative Health Task Force - Independent Expert Panel, 1996 (revised 2002 Nov), Rockville, MD

3. Welch HG. Should I be tested for cancer? Maybe not and here's why. Berkley, CA: The University of California Press, 2004

4. McGlynn EA, Asch SM, Adams J, et al. The quality of health care delivered to adults in the United States. N Engl J Med 2003;348:2635-2645.

5. Yarnall KS, Pollak KI, Ostbye T, et al. Primary care: is there enough time for prevention? Am J Public Health 2003;93(4):635-641. 